\title{
Incentive-Compatible in Dominant Strategies Mechanism Design for an Assembler under Asymmetric Information
}

\author{
Zhaolin Li \\ Discipline of Business Analytics \\ The University of Sydney Business \\ School, NSW 2006, Australia \\ Telephone: 61291140751 \\ Fax: 61293516409 \\ erick.li@sydney.edu.au \\ Jennifer K. Ryan \\ College of Business \\ University of Nebraska - Lincoln \\ Lincoln, NE 68588 \\ Telephone: 402-472-2256 \\ jennifer.ryan@unl.edu \\ Corresponding Author \\ Lusheng Shao \\ Department of Management and Marketing \\ Faculty of Business and Economics \\ The University of Melbourne \\ Melbourne, Australia \\ Telephone: 61383444558 \\ Fax: 61393494293 \\ lusheng.shao@unimelb.edu.au \\ Daewon Sun \\ Department of IT, Analytics, and Operations \\ Room 359, Mendoza College of Business \\ Notre Dame, IN 46556 \\ Telephone: 574-631-0982 \\ Fax: 574-631-5127 \\ dsun@nd.edu
}




\title{
Incentive-Compatible in Dominant Strategies Mechanism Design for an Assembler under Asymmetric Information
}

\begin{abstract}
Assembly systems, in which various components are sourced from multiple suppliers and assembled into the final product, which is sold to external customers, are found in a variety of industries. In many practical settings, the assembler possesses incomplete information regarding the marginal cost of each supplier. This lack of complete information poses a challenge for the assembler in designing contract mechanisms. In this paper, we investigate the assembler's contract design problem by proposing a contracting mechanism that can significantly outperform an alternative mechanism that was previously presented in the literature, especially when the uncertainty regarding customer demand is significant. Our mechanism is incentive compatible in dominating strategies (ICDS) and maximizes the assembler's expected profit while ensuring that every supplier truthfully reveals their own production cost, regardless of how the other suppliers might behave. In this ICDS mechanism, the assembler orders the same number of components from each supplier. This "balanced ordering" property does not hold for the alternative mechanism from the literature. Finally, to simplify the proposed ICDS mechanism, we introduce a hybrid mechanism, under which the complexity of the contract offered to a given supplier depends on the importance of that supplier to the assembler's overall profit. We conduct a set of numerical experiments to demonstrate that, in many cases, this proposed hybrid mechanism provides performance close to that of the optimal mechanism, and can significantly outperform the alternative mechanism from the literature.
\end{abstract}

Keywords: Procurement, Assembly Systems, Contract Design, Incentive Compatible in Dominating Strategies.

Received: March 2017; accepted: June 2018 by Eric Johnson after three revisions 


\section{Introduction}

Assembly production systems can be found in a wide variety of industries, including automotive, heavy equipment, and consumer electronics manufacturing. In these industries, assemblers will often outsource the production of the components in order to capitalize on the suppliers' technical expertise and to take advantage of the economies-of-scale in the suppliers' production processes. However, these component suppliers may not always be willing to share their cost information with the assembler, out of concern that the assembler may take advantage of such information to reduce the payment to the supplier. Further, multi-national enterprises (MNEs) often procure components from hundreds of suppliers across the globe. Inevitably, a number of these suppliers will be first-time trading partners of the MNE, implying that the MNE will have limited information regarding those suppliers' costs. Even when the relationship with the trading partner is longstanding, suppliers will often experience fluctuations in raw material costs, due to volatility in commodity markets (Levy and Ferazani, 2006), which would make any cost data obtained by the assembler during the previous contracting cycles no longer valid. For these reasons, an important question is how an assembler should best design the contracts offered to the suppliers in order to cope with information asymmetry regarding the suppliers' marginal production costs, particularly in settings with uncertain demand for the final product.

The literature discusses a number of different contracting mechanisms that can be used by an assembler in settings with asymmetric information (see Section 1.2 for more details). For example, Fang et al. (2014), hereafter referred to as FRW, derive a mechanism in which the contract offered to each supplier depends only on that supplier's cost, and not on the costs of the other suppliers. Thus, the mechanism is straightforward to understand and to implement, even in settings with a very large number of suppliers. However, the resulting order quantities may not be balanced, i.e., the assembler may order a different number of components from each supplier. Such an unbalanced ordering policy is clearly inefficient and can potentially lead to significant waste, in the sense that it may result in more unused components.

Motivated by this observation, in this paper we propose an alternative contracting mechanism (the incentive compatible in dominating strategies, or ICDS, mechanism) in which the assembler 
offers the suppliers a menu of contracts under which the payments and procurement quantities are contingent on the reported costs of all suppliers. Given this menu, each supplier independently reports their marginal cost. After receiving the reports from all suppliers, the assembler determines the procurement quantity and payment for each supplier, according to the contract menu that was previously announced. While the ICDS mechanism is clearly more complex to compute and implement than the FRW mechanism, particularly when the number of suppliers is large, we will demonstrate that balanced ordering, under which the assembler orders the same number of components from each supplier, is always optimal under the ICDS mechanism, and that the ICDS mechanism will always perform at least as well as the FRW mechanism.

Practical settings often require assemblers to contract with a large number of suppliers, where not all suppliers are of equal importance to, or have equal impact on, the assembler's profit. However, when the number of suppliers is moderate to large, the ICDS mechanism becomes too complex to implement, while the FRW mechanism offers poor performance. To address this issue, we propose an alternative hybrid mechanism, under which the assembler employs the ICDS mechanism only for the most important suppliers, using a simpler mechanism for the remaining suppliers. Using a numerical study, we will demonstrate that this hybrid mechanism can perform quite well relative to the ICDS mechanism and can significantly outperform the FRW mechanism, particularly when (1) there is significant uncertainty regarding the suppliers' costs, (2) there is significant uncertainty regarding the end-customer demand, and (3) the number of suppliers is large.

The analysis of the alternative contracting mechanisms (the ICDS, FRW, and hybrid mechanisms) contained in this paper provides important practical guidance for assemblers regarding when the use of more complex contracting mechanisms, such as the ICDS mechanism, can be warranted. For example, we will demonstrate that using the ICDS mechanism adds the most value for the assembler, compared to both the hybrid and FRW mechanisms, when there is significant uncertainty regarding the end-product demand. In other words, when demand uncertainty is significant, it is important for the assembler to use a mechanism in which the contract parameters are dependent on all suppliers' costs. By doing so, the assembler can make use of as much information as possible regarding the suppliers' costs when setting the order quantity. We also find that more complex (and hence more sophisticated) mechanisms are necessary when the assembler has significant un- 
certainty regarding the suppliers' costs. Thus, a key insight obtained from this research is that while simplicity and ease of implementation are of practical importance, in complex settings with information asymmetry, they should not be the only objective in contract design.

\section{$1.1 \quad$ Literature Review}

In this paper, we consider an assembler who must contract with a set of suppliers who possess private information regarding their marginal production costs. The contracts specify the quantity that will be provided by each supplier, as well as the payment from the assembler. For this setting, we propose an incentive compatible in dominating strategies (ICDS) mechanism (Bergemann and Välimäki, 2006), designed to maximize the assembler's profit, while ensuring that every supplier truthfully reports their cost, regardless of whether the other suppliers report truthfully. In the literature on mechanism design (Section 2.1 of Bergemann and Välimäki, 2006 and Jehiel et al. 2006), there are three main types of incentive compatible (IC) mechanisms: Bayesian incentive compatible (BIC), ex post incentive compatible (EPIC), and incentive compatible in dominating strategies (ICDS). In a BIC mechanism, each supplier uses the (publicly available) joint distribution of the suppliers' marginal costs to evaluate the expected utility associated with different reporting strategies. The BIC mechanism requires that each supplier obtains the highest expected utility by truthfully reporting his cost, given that all other suppliers also report truthfully. In an EPIC mechanism, each supplier prefers truth-telling even after knowing the other suppliers' reported costs (which are assumed to be truthful). In an ICDS mechanism, each supplier prefers truthtelling regardless of whether the other suppliers' report truthfully. Of these three mechanisms, the ICDS constraints are the most stringent, while the BIC constraints are the weakest (Chung and Ely 2007), where weaker constraints imply (weakly) higher expected profit for the assembler.

The main criticism of BIC mechanisms (e.g., Wilson 1987) is the assumption that the joint distribution the suppliers' marginal costs is publicly known. In practice, assemblers often source from a geographically-dispersed set of suppliers, who may have little information regarding each

other's cost structure. Thus, the assumption that the joint distribution is known to all suppliers is unlikely to hold. To overcome this difficulty, researchers consider either EPIC or ICDS mechanisms, which do not require the joint distribution to be public information. The key difference between 
the EPIC and ICDS mechanisms is subtle. In an ICDS mechanism, each supplier ignores the other suppliers' reporting strategies. In contrast, in an EPIC mechanism, each supplier assumes that other suppliers truthfully report their costs. In settings with private valuations (which holds in our model since, given the payments and quantities, a supplier's profit depends only on his own type), the EPIC and ICDS mechanisms are equivalent (Chung and Ely 2007).

There is previous work in the economics literature considering ICDS mechanisms, which are sometimes referred to as dominant-strategy incentive-compatibility (DSIC) mechanisms. A formal definition of the ICDS mechanism can be found in Definition 2 of Bergemann and Valimaki (2006). ICDS mechanisms, which do not require that each supplier knows the joint probability distribution for the suppliers' unit costs, are often discussed in the context of Wilson's (1987) criticism of the BIC mechanisms' overreliance on common knowledge assumptions. Bergemann and Morris (2005) and Chung and Ely (2006) develop theoretical motivations for the ICDS mechanism. In addition, there is a stream of the economics literature considering the relative performance of the ICDS and BIC mechanisms. See Appendix B for a discussion of this literature, as well as a comparison of the ICDS and BIC mechanisms for our assembly setting.

While ICDS mechanisms have been well-studied in economics, they have not been applied in the operations management literature (with the exception of Chaturvedi and Martinez-de-Albeniz, 2011, as discussed below). In addition, our approach to the model and analysis differs from that generally used in the economics literature. For example, the problem considered in Section 3 of Melumad, et al. (1995) has some similarity to our model. However, that previous work does not explicitly consider issues specific to the assembly setting, such as demand uncertainty and the question of whether the order quantities are balanced. Further, that previous work considers just two agents (suppliers) with independent costs (and hence the authors find that the ICDS and BIC mechanisms are equivalent). In summary, to the best of our knowledge, our paper is among the first to study ICDS mechanisms for an assembly setting with asymmetric information regarding the suppliers' costs. In doing so, we take a different perspective from the economics literature, as discussed above, by focusing on explicitly characterizing the optimal ICDS procurement mechanism for the assembler, including both payments and order quantities, in the context of a general number of suppliers and uncertain demand for the final product. We also allow the suppliers costs to be 
generally correlated, a setting which has not been thoroughly studied in the economics literature.

Besides FRW, the most closely-related work to our paper is Hu and Qi (2017), who also study mechanism design for an assembler under asymmetric information regarding the suppliers' costs, but under the BIC solution framework. Their model assumes that the customer demand is linear in price, the final product requires just two components (i.e., there are just two suppliers), and the marginal cost of each supplier is independently-distributed. As discussed in Appendix B, the BIC mechanism can in some cases outperform an ICDS mechanism. However, the use of an ICDS mechanism allows us to consider a general number of suppliers (components), as well as any degree of correlation between the suppliers' costs. In addition, because our ICDS mechanism does not require the assumption that all suppliers know the joint probability distribution of the marginal costs, our setting is more practical (robust) than that considered in $\mathrm{Hu}$ and Qi (2017). Furthermore, by considering uncertain demand, we are able to provide insights into the impact of this uncertainty on the assembler's optimal decisions.

Finally, while there exists a large body of supply chain management literature considering contractual mechanisms under asymmetric information, the majority of that literature involves a single agent and a single component. In contrast, our model considers multiple agents (suppliers) and multiple components. There are a few previous works that consider settings with multiple agents and/or components. Kayis et al. (2013) consider a two-agent model in which the tier-2 agent supplies the semi-finished good to the tier-1 agent and then the tier-1 agent supplies the finished good to the buyer. The agents possess private cost information and the buyer assesses the benefit of delegating procurement to the tier-1 agent. While Kayis et al. (2013) consider a serial supply chain, our model studies an assembly supply chain. Yang et al. (2012) examine the benefit of allowing two suppliers, who possess private information regarding their disruption risks, to compete. While the goods produced by the two suppliers in Yang et al. (2012) are substitutes, in our model the goods are complements. Finally, Chaturvedi and Martínez-de-Albéniz (2011) develop an ICDS procurement mechanism for a setting in which multiple suppliers possess private information regarding their cost and reliability. However, they consider substitute goods in an auction setting, while our model considers complementary goods in an assembly system. 


\subsection{Managerial Insights and Contributions}

We conclude this section with a summary of the insights and contributions provided by this paper.

- We derive the optimal ICDS mechanism for an assembler and demonstrate that this optimal mechanism always exists, which implies that an ICDS mechanism can be used by an assembler to cope with asymmetric information regarding her suppliers' marginal costs.

- We prove that balanced ordering is optimal under the ICDS mechanism. This is in contrast to the unbalanced ordering result for the FRW mechanism. The optimality of balanced ordering is particularly important in assembly industries such as consumer electronics, where component overstocking can be quite costly due to obsolescence.

- We demonstrate that the ICDS mechanism always outperforms the FRW mechanism, which can be ineffective in extracting cost information from the suppliers when the number of suppliers is greater than two, and we characterize the conditions under which the ICDS mechanism offers the most value relative to the FRW mechanism.

- We find that the value of using the ICDS mechanism increases with the uncertainty in demand due to the fact that, in contrast to the FRW mechanism, the ICDS mechanism allows the buyer to make quantity decisions using information on all of the suppliers' costs. Since many assemblers operate in settings with significant demand uncertainty (e.g., in the consumer electronics industry), this result provides an important insight that can assist assemblers in managing their procurement.

- We develop and propose an easier-to-implement hybrid mechanism which effectively balances complexity of implementation and performance, enhancing performance relative to the simpler FRW mechanism, while offering reduced complexity relative to the ICDS mechanism.

The rest of the paper is organized as follows. Section 2 derives the optimal ICDS mechanism for a setting with a general number of suppliers and binary types, and presents an easy-to-implement hybrid mechanism. Section 3 presents the results of a numerical study in which we demonstrate that the ICDS mechanism can significantly outperform the FRW mechanism, and evaluate the performance of the proposed hybrid mechanism. Section 4 extends the basic model to a setting with continuous types. Section 5 concludes the analysis. All proofs can be found in Appendix A. All appendices can be found in the online supplement.

\section{Assembler's Problem: Formulation and Analysis}

In this paper, we consider a model in which an assembler acts as an uninformed principal, while the $n$ suppliers act as the informed agents who possess private information regarding the marginal 
production cost for the component that they produce. We refer to the assembler as "she" and to each of the suppliers as "he." We assume that each unit of final product requires one unit of each of the $n$ components. Each supplier produces a single type of component and contracts with the assembler to provide a certain quantity of that component in exchange for a payment. We first characterize the optimal ICDS mechanism when the suppliers' types (production costs) are binary. In Section 4, we extend the analysis to consider a continuous distribution for the suppliers' types.

\subsection{ICDS Model Formulation}

We consider an assembler who sells a product that is assembled from $n \geq 2$ distinct components. The final product is sold at a fixed selling price of $r$ per unit, and requires (without loss of generality) one unit of each component. The external demand for the final product, denoted by $D$, is a non-negative random variable with cumulative distribution function $(\mathrm{CDF})$ denoted by $F(\cdot)$ and probability density function $(\mathrm{PDF})$ denoted by $f(\cdot)$. Prior to observing the final product demand, the assembler procures components from the $n$ different suppliers, indexed by $i \in\{1,2, \ldots, n\}$, where supplier $i$ produces only component $i$. The assembler's cost to assemble the final product is normalized to zero. We define $S(q)=r E[\min (D, q)]$ as the expected sales revenue when $q$ units of the final product can be assembled. The first derivative of $S(q)$ with respect to $q$ is $S^{\prime}(q)=r(1-F(q))$ and the second derivative is $S^{\prime \prime}(q)=-r f(q)<0$. After observing the external customer demand, the assembler uses the available components to assemble the final product. Any unsatisfied demand is lost and any unused components are salvaged with zero value.

The assembler knows that supplier $i$ may be either a low-cost type, denoted by type- $l$, or a high-cost type, denoted by type- $h$. We use $t_{i} \in\{l, h\}$ to represent the type of supplier $i$. If supplier $i$ is type- $t_{i}$, his production cost is $c_{i}\left(t_{i}\right)>0$ per unit. For supplier $i$, we use $\Delta_{i}=c_{i}(h)-c_{i}(l)>0$ to denote the cost difference between the high-cost type and the low-cost type. There are $2^{n}$ possible combinations of the suppliers' types. We use bold-face variables to represent vectors. Let $\mathbf{t}=\left(t_{1}, t_{2}, \ldots, t_{n}\right)$ denote the vector of all supplier types and $\mathbf{t}_{-i}=\left(t_{1}, \ldots, t_{i-1}, t_{i+1}, \ldots, t_{n}\right)$ denote the vector of all types excluding $t_{i}$. Let $\beta(\mathbf{t})$ denote the joint probability that the vector of suppliers' types is $\mathbf{t}$. Let $\beta\left(t_{i} \mid \mathbf{t}_{-i}\right)$ denote the conditional probability that supplier $i$ is type $t_{i}$ when the vector of the other $n-1$ suppliers' types is $\mathbf{t}_{-i}$. For any given $\mathbf{t}$, we define $c(\mathbf{t})=\sum_{i=1}^{n} c_{i}\left(t_{i}\right)$ as 
the total cost to produce one unit of the final product. We assume that $c(\mathbf{h}) \equiv \sum_{i=1}^{n} c_{i}(h)<r$, which ensures that the assembler always produces a positive amount of the final product.

The assembler announces a procurement mechanism (i.e., a contract menu) and asks each supplier to report their marginal cost of production accordingly. The revelation principle states that it suffices for the assembler to only consider mechanisms that are direct and incentive-compatible (Myerson 1979). A mechanism specifies a message space for each supplier $i$. After observing his type to be $t_{i}$, supplier $i$ chooses to report a message from that message space to the assembler. A mechanism is called a direct mechanism if the message space is the same as the supplier's type space. With binary types, the direct message space is thus $\{l, h\}$ for all $i$. As discussed in detail below, in an incentive-compatible mechanism, each supplier finds it beneficial to truthfully report his true type, i.e., supplier $i$ with type $t_{i}$ reports the message $t_{i}$.

After receiving the reported types from all $n$ suppliers, $\mathbf{t}=\left(t_{1}, t_{2}, \ldots, t_{n}\right)$, the assembler procures $q_{i}(\mathbf{t})$ units of component $i$ from supplier $i$ and makes a payment of $P_{i}(\mathbf{t})$ to supplier $i$. The payments and procurement quantities are contingent on the reported types of all suppliers. In contrast, the FRW mechanism takes the form $\left\{P_{i}\left(t_{i}\right), q_{i}\left(t_{i}\right)\right\}$. Therefore, the FRW mechanism could be derived by adding the following constraints into our model: $P_{i}\left(t_{i}, \mathbf{t}_{-i}\right)=P_{i}\left(t_{i}\right)$ and $q_{i}\left(t_{i}, \mathbf{t}_{-i}\right)=q_{i}\left(t_{i}\right)$. Thus, the mechanism developed in this paper always provides the assembler with an expected profit at least as high as that provided by the FRW mechanism. Further, in Section 3 we demonstrate that these extra constraints in the FRW mechanism can significantly reduce the assembler's profit.

Without loss of generality, we divide the payment to supplier $i, P_{i}(\mathbf{t})$, into two parts such that

$$
P_{i}(\mathbf{t})=w_{i}(\mathbf{t})+c_{i}\left(t_{i}\right) q_{i}(\mathbf{t})
$$

where $w_{i}(\mathbf{t})$ represents the markup, $c_{i}\left(t_{i}\right)$ is supplier $i$ 's reported cost per unit, and $q_{i}(\mathbf{t})$ is the procurement quantity. Thus, $c_{i}\left(t_{i}\right) q_{i}(\mathbf{t})$ is the total production cost incurred by supplier $i$, based on supplier $i$ 's reported cost. In other words, each contract in the ICDS mechanism consists of a fixed payment (the markup), a variable (per unit) payment, which is the same as the supplier's reported per unit cost, and a quantity $q_{i}(\mathbf{t})$, which depends on the reported types of all suppliers. Finally, we normalize the reservation value of each supplier to be zero.

Let $\mathbf{q}(\mathbf{t})=\left(q_{1}(\mathbf{t}), q_{2}(\mathbf{t}), \ldots, q_{n}(\mathbf{t})\right)$ be the vector of procurement quantities if the vector of 
reported types is $\mathbf{t}$. The assembler's expected profit, denoted by $Z$, can be written as the total supply chain expected profit, minus any rents paid to the suppliers:

$$
Z=\sum_{\mathbf{t}} \beta(\mathbf{t})[\underbrace{S(\min (\mathbf{q}(\mathbf{t})))-\sum_{i=1}^{n} c_{i}\left(t_{i}\right) q_{i}(\mathbf{t})}_{\text {Total profit of the supply chain }}-\underbrace{\sum_{i=1}^{n} w_{i}(\mathbf{t})}_{\text {Total rents }}]
$$

As discussed next, the model constraints will ensure that all suppliers truthfully report their types.

As described in Appendix B, there are a number of different types of incentive-compatible mechanisms. We focus on designing a mechanism that is incentive-compatible in dominant strategies (ICDS). Under such a mechanism, each supplier finds it optimal to truthfully report their type, regardless of what the other suppliers report. If the suppliers' true types are given by $\mathbf{t}$ and if each supplier reports truthfully, $w_{i}(\mathbf{t})$ represents the information rent (or the surplus) earned by supplier $i$. However, if supplier $i$, whose true type is $t_{i}$, misrepresents his private cost information by reporting his type to be $t_{i}^{\prime} \neq t_{i}$, then supplier $i$ may suffer a cost overrun if he understates his true cost, or achieve a cost reduction if he overstates his true cost. To ensure that each supplier $i$ prefers truth-revealing regardless of what other suppliers might report, when supplier $i$ is of type $t_{i}$, the ICDS constraints specify that the payments must satisfy

$$
P_{i}\left(t_{i}, \mathbf{t}_{-i}\right)-c_{i}\left(t_{i}\right) q_{i}\left(t_{i}, \mathbf{t}_{-i}\right) \geq P_{i}\left(t_{i}^{\prime}, \mathbf{t}_{-i}\right)-c_{i}\left(t_{i}\right) q_{i}\left(t_{i}^{\prime}, \mathbf{t}_{-i}\right)
$$

for any $i, t_{i} \neq t_{i}^{\prime}$, and $\mathbf{t}_{-i}$. Using (1), constraints (3) can be written as

$$
w_{i}\left(t_{i}, \mathbf{t}_{-i}\right) \geq w_{i}\left(t_{i}^{\prime}, \mathbf{t}_{-i}\right)+\left[c_{i}\left(t_{i}^{\prime}\right)-c_{i}\left(t_{i}\right)\right] q_{i}\left(t_{i}^{\prime}, \mathbf{t}_{-i}\right)
$$

for any $i, t_{i} \neq t_{i}^{\prime}$, and $\mathbf{t}_{-i}$. Notice that these constraints must hold for any $\mathbf{t}_{-i}$, i.e., regardless of the other suppliers' (reported) types.

The right-hand-side (RHS) of (4) indicates that if supplier- $i$ misrepresents his cost to be $c_{i}\left(t_{i}^{\prime}\right) \neq c_{i}\left(t_{i}\right)$, the assembler would change the procurement quantity from $q_{i}\left(t_{i}, \mathbf{t}_{-i}\right)$ to $q_{i}\left(t_{i}^{\prime}, \mathbf{t}_{-i}\right)$. Given that constraint (4) is satisfied, a type- $t_{i}$ supplier- $i$ receives a surplus (rent) of $w_{i}(\mathbf{t})$ in the equilibrium in which truth-revealing is the dominating strategy. Thus, the individual rationality 
(IR) constraints, which guarantee that the suppliers earn a non-negative surplus in the equilibrium, can be written as $w_{i}(\mathbf{t}) \geq 0$.

The ICDS constraints (4) must hold for any type for supplier $i$. For example, if supplier $i$ is type- $l$, then $t_{i}=l$ and $t_{i}^{\prime}=h$, and (4) becomes $w_{i}\left(l, \mathbf{t}_{-i}\right) \geq w_{i}\left(h, \mathbf{t}_{-i}\right)+\left[c_{i}(h)-c_{i}(l)\right] q_{i}\left(h, \mathbf{t}_{-i}\right)$. On the other hand, if $t_{i}=h$ and $t_{i}^{\prime}=l$, then (4) becomes $w_{i}\left(h, \mathbf{t}_{-i}\right) \geq w_{i}\left(l, \mathbf{t}_{-i}\right)+\left[c_{i}(l)-c_{i}(h)\right] q_{i}\left(l, \mathbf{t}_{-i}\right)$. Since the type of supplier $i$ is not known, both of these ICDS constraints must hold. If we add these constraints together and re-arrange terms, we have that $\left[q_{i}\left(h, \mathbf{t}_{-i}\right)-q_{i}\left(l, \mathbf{t}_{-i}\right)\right]\left[c_{i}(h)-c_{i}(l)\right] \leq 0$, or, alternatively, $\left[q_{i}\left(l, \mathbf{t}_{-i}\right)-q_{i}\left(h, \mathbf{t}_{-i}\right)\right]\left[c_{i}(l)-c_{i}(h)\right] \leq 0$. In other words, for any $t_{i}, t_{i}^{\prime} \in\{l, h\}, t_{i} \neq t_{i}^{\prime}$, and any $\mathbf{t}_{-i}$, we have the following constraint, which we refer to as the feasibility constraint:

$$
\left[c_{i}\left(t_{i}\right)-c_{i}\left(t_{i}^{\prime}\right)\right]\left[q_{i}\left(t_{i}, \mathbf{t}_{-i}\right)-q_{i}\left(t_{i}^{\prime}, \mathbf{t}_{-i}\right)\right] \leq 0,
$$

The procurement quantities for a feasible mechanism must satisfy (5). Thus, if a supplier reports a higher cost, i.e., if a type- $t_{i}$ supplier $i$ reports the type $t_{i}^{\prime}$, with $c_{i}\left(t_{i}^{\prime}\right)>c_{i}\left(t_{i}\right)$, the assembler must procure fewer components from this supplier, i.e., $q_{i}\left(t_{i}^{\prime}, \mathbf{t}_{-i}\right) \leq q_{i}\left(t_{i}, \mathbf{t}_{-i}\right)$, with all else unchanged.

In summary, the assembler's optimization problem is to maximize (2) subject to (4) and the IR constraints, i.e., $w_{i}(\mathbf{t}) \geq 0$. Before we discuss the solution to this problem, we next consider the solutions of two benchmark mechanisms.

\subsection{Benchmark Mechanisms}

As noted above, we assume $c(\mathbf{h})=\sum_{i=1}^{n} c_{i}(h)<r$. In this case, a feasible solution for the assembler is to apply a naïve mechanism, defined below, in which she assumes that every supplier is the highcost type. Under the naïve mechanism, all suppliers agree to the contracts offered by the assembler, with the low-cost suppliers earning a positive rent, and the high-cost suppliers earning zero rent.

Definition 1 In the naïve mechanism, the assembler procures $q_{0}$ units of component $i$ from supplier $i$ with payment $c_{i}(h) q_{0}$, where $q_{0}$ satisfies $F\left(q_{0}\right)=\frac{r-c(\mathbf{h})}{r}$, i.e., $q_{0}$ is the newsvendor solution when the selling price is $r$ and the procurement cost is $c(\mathbf{h})$. The assembler's expected profit is $Z_{0}=S\left(q_{0}\right)-c(\mathbf{h}) q_{0}$, while the surplus (or rent) earned by a type- $t_{i}$ supplier $i$ is $\left[c_{i}(h)-c_{i}\left(t_{i}\right)\right] q_{0}$. 
It is also useful to consider the assembler's solution when full information is available, i.e., when she is aware of all suppliers' costs. In this case, the assembler's expected profit can be written as

$$
Z=\sum_{\mathbf{t}} \beta(\mathbf{t})\left[S(\min (\mathbf{q}(\mathbf{t})))-\sum_{i=1}^{n} c_{i}\left(t_{i}\right) q_{i}(\mathbf{t})\right],
$$

where $\mathbf{t}$ is the vector of the suppliers' true costs. It is straightforward to show that the fullinformation order quantities are balanced, i.e., $q_{1}(\mathbf{t})=q_{2}(\mathbf{t})=\ldots=q_{n}(\mathbf{t})$. Therefore, the

optimal balanced procurement quantity, denoted by $\bar{q}(\mathbf{t})$, satisfies $F(\bar{q}(\mathbf{t}))=\frac{r-c(\mathbf{t})}{r}$, where $c(\mathbf{t})=$ $\sum_{i=1}^{n} c_{i}\left(t_{i}\right)$, and the assembler's expected profit with full information is $\bar{Z}$, where

$$
\bar{Z}=\sum_{\mathbf{t}} \beta(\mathbf{t})[S(\bar{q}(\mathbf{t}))-c(\mathbf{t}) \bar{q}(\mathbf{t})]
$$

\subsection{Relaxed Problem Definition and Solution}

To derive the optimal mechanism, we first omit some constraints. We then find a solution to the relaxed problem, i.e., the problem in which we omit some constraints, and check whether the relaxed solution satisfies the omitted constraints. If so, we have the optimal solution to the full problem.

The assembler's problem is to maximize (2) subject to (4) and the IR constraints, $w_{i}(\mathbf{t}) \geq 0$. With two possible types $(l$ and $h)$ for each supplier, we can divide the constraints (4) into two sets. The downward IC constraints ensure that the low-type suppliers truthfully report their type, i.e.,

$$
w_{i}\left(l, \mathbf{t}_{-i}\right) \geq w_{i}\left(h, \mathbf{t}_{-i}\right)+\Delta_{i} q_{i}\left(h, \mathbf{t}_{-i}\right),
$$

where $\Delta_{i}=c_{i}(h)-c_{i}(l)>0$. Similarly, the upward IC constraints ensure that the high-type suppliers truthfully report their type, i.e.,

$$
w_{i}\left(h, \mathbf{t}_{-i}\right) \geq w_{i}\left(l, \mathbf{t}_{-i}\right)-\Delta_{i} q_{i}\left(l, \mathbf{t}_{-i}\right) .
$$

Because the RHS of the upward constraint (9) contains a negative term, i.e., $-\Delta_{i} q_{i}\left(l, \mathbf{t}_{-i}\right)$, we conjecture that this constraint is easier to satisfy than the downward constraint (8). Therefore, we define the relaxed problem as the assembler's problem, but with all of the upward constraints (9) omitted (see Appendix E). For this relaxed problem, we have the following result: 
Lemma 1 In the relaxed problem in which constraints (9) are removed, for any given $\mathbf{t}$, the optimal procurement quantities are balanced, i.e., they satisfy $q_{1}(\mathbf{t})=q_{2}(\mathbf{t})=\ldots=q_{n}(\mathbf{t})$.

This balanced order quantity result (which, as will be seen, also holds for the optimal ICDS mechanism) stands in contrast to the result in FRW. The reason for this difference is that our mechanism, unlike the FRW mechanism, allows the assembler to determine the procurement quantities based on all suppliers' reported types. Thus, we find that the nature of the mechanism not only affects the assembler's profits, but also the resulting order quantities, with the ICDS mechanism providing the additional benefits associated with balanced order quantities, such as reduced waste.

We can now drop the subscript $i$ and use $q(\mathbf{t})$ to represent the order quantity given the types, t, for the relaxed problem. The feasibility constraint (5) thus becomes

$$
q\left(l, \mathbf{t}_{-i}\right) \geq q\left(h, \mathbf{t}_{-i}\right), \text { for any } \mathbf{t}_{-i},
$$

which indicates that when one particular supplier reports a high cost, the quantity supplied by all suppliers weakly decreases.

\subsubsection{Optimal Rents for the Relaxed Problem}

We next consider how to set the rents for the relaxed problem. Suppose the order quantities, $q(\mathbf{t})$, are feasible and exogenously specified. The assembler's expected sales revenue equals $S(q(\mathbf{t}))$. Given the order quantities, the assembler seeks to minimize the total expected rents paid to the suppliers, subject to (8), which implies that all of the downward IC constraints (which have not been relaxed) must be binding at the optimal solution. Therefore, we have the following result:

Lemma 2 Given $q(\mathbf{t})$, the optimal rents for the relaxed problem are:

$$
w_{i}\left(l, \mathbf{t}_{-i}\right)=\Delta_{i} q\left(h, \mathbf{t}_{-i}\right) \text { and } w_{i}\left(h, \mathbf{t}_{-i}\right)=0, \text { for any } \mathbf{t}_{-i} .
$$

If supplier $i$ is high-cost, he receives zero rent, regardless of the other suppliers' types. However, if supplier $i$ is low-cost, he receives positive information rent equal to the cost reduction he would achieve if he mimics a high-cost supplier. 
We can now rewrite the assembler's expected profit. Let $L(\mathbf{t})=\left\{i \mid t_{i}=l\right\}$ be the set of suppliers that are low-cost when the vector of types is $\mathbf{t}$ and let $H(\mathbf{t})=\left\{i \mid t_{i}=h\right\}$ be the set of suppliers that are high-cost. Substituting the optimal rents in (11) into the assembler's profit in (2), we can write the assembler's profit for the relaxed problem, denoted by $Z_{R}$, given $q(\mathbf{t})$, as follows:

$$
\begin{aligned}
Z_{R} & =\sum_{\mathbf{t}} \beta(\mathbf{t})\left[S(q(\mathbf{t}))-c(\mathbf{t}) q(\mathbf{t})-\sum_{i=1}^{n} w_{i}(\mathbf{t})\right] \\
& =\sum_{\mathbf{t}} \beta(\mathbf{t}) S(q(\mathbf{t}))-\sum_{\mathbf{t}} \beta(\mathbf{t})\left[c(\mathbf{t}) q(\mathbf{t})+\sum_{i=1}^{n} w_{i}(\mathbf{t})\right] \\
& =\sum_{\mathbf{t}} \beta(\mathbf{t}) S(q(\mathbf{t}))-\sum_{\mathbf{t}} \beta(\mathbf{t})\left[c(\mathbf{t}) q(\mathbf{t})+\sum_{i \in L(\mathbf{t})} \Delta_{i} q\left(h, \mathbf{t}_{-i}\right)\right] \\
& =\sum_{\mathbf{t}} \beta(\mathbf{t}) S(q(\mathbf{t}))-\sum_{\mathbf{t}} q(\mathbf{t})\left[\beta(\mathbf{t}) c(\mathbf{t})+\sum_{i \in H(\mathbf{t})} \Delta_{i} \beta\left(l, \mathbf{t}_{-i}\right)\right] \\
& =\sum_{\mathbf{t}} \beta(\mathbf{t}) S(q(\mathbf{t}))-\sum_{\mathbf{t}} \beta(\mathbf{t}) q(\mathbf{t})\left[c(\mathbf{t})+\frac{1}{\beta(\mathbf{t})} \sum_{i \in H(\mathbf{t})} \Delta_{i} \beta\left(l, \mathbf{t}_{-i}\right)\right] \\
& =\sum_{\mathbf{t}} \beta(\mathbf{t})\left[S(q(\mathbf{t}))-q(\mathbf{t})\left(c(\mathbf{t})+\frac{1}{\beta(\mathbf{t})} \sum_{i \in H(\mathbf{t})} \Delta_{i} \beta\left(l, \mathbf{t}_{-i}\right)\right]\right.
\end{aligned}
$$

In (12), the third step replaces the rents, $w_{i}(\mathbf{t})$, with their optimal values, as defined in (11). Since $w_{i}\left(h, \mathbf{t}_{-i}\right)=0$, for any $\mathbf{t}_{-i}$, i.e., the optimal rent earned by any high-cost supplier is equal to 0 , in the third step, rather than summing the optimal rents over all $i$, we sum only for the low-cost suppliers, i.e., we sum over $i \in L(\mathbf{t})$. The fourth step groups terms according to the $q(\mathbf{t})$ they involve. Notice that, in the third step, the order quantities, i.e., $q\left(h, \mathbf{t}_{-i}\right)$, are for the case in which supplier $i$ is high-cost. Thus, the sum in the final term of the fourth step of (12) is over $i \in H(\mathbf{t})$. Finally, to make this derivation more clear, we provide Example 1 below which demonstrates the derivation of (12) for a setting with two suppliers.

For expositional simplicity, we define the virtual cost, $v(\mathbf{t})$, as follows:

$$
v(\mathbf{t})=c(\mathbf{t})+\frac{1}{\beta(\mathbf{t})} \sum_{i \in H(\mathbf{t})} \Delta_{i} \beta\left(l, \mathbf{t}_{-i}\right) .
$$


Using equation (13), we can re-write the assembler's profit, $Z_{R}$, given $q(\mathbf{t})$, as follows:

$$
Z_{R}=\sum_{\mathbf{t}} \beta(\mathbf{t})[S(q(\mathbf{t}))-v(\mathbf{t}) q(\mathbf{t})]
$$

To illustrate these results, we provide the following example.

Example 1 Consider a case with $n=2$ suppliers. We have $H(l, l)=\emptyset, H(l, h)=\{2\}, H(h, l)=$ $\{1\}$, and $H(h, h)=\{1,2\}$. Using (13), we find that the virtual costs are as follows:

$$
\left\{\begin{array}{l}
v(l, l)=c(l, l), \\
v(l, h)=c(l, h)+\frac{\beta(l, l)}{\beta(l, h)} \Delta_{2}, \\
v(h, l)=c(h, l)+\frac{\beta(l, l)}{\beta(h, l)} \Delta_{1}, \\
v(h, h)=c(h, h)+\frac{\beta(l, h)}{\beta(h, h)} \Delta_{1}+\frac{\beta(h, l)}{\beta(h, h)} \Delta_{2} .
\end{array}\right.
$$

We now explain how to derive these virtual costs and optimal rents by using the following downward IC constraints, obtained from (8):

$$
\left\{\begin{array}{l}
w_{1}(l, l) \geq w_{1}(h, l)+\Delta_{1} q(h, l), \\
w_{2}(l, l) \geq w_{2}(l, h)+\Delta_{2} q(l, h), \\
w_{1}(l, h) \geq w_{1}(h, h)+\Delta_{1} q(h, h), \\
w_{2}(h, l) \geq w_{2}(h, h)+\Delta_{1} q(h, h) .
\end{array}\right.
$$

The assembler seeks to minimize the total expected rents. Because $w_{1}(h, l)$ only appears on the RHS of the first downward constraint, its optimal value must be zero. This result implies that $w_{1}(l, l)=\Delta_{1} q(h, l)$. Similarly, we find that the other optimal rents satisfy $w_{2}(l, l)=\Delta_{2} q(l, h)$, $w_{1}(l, h)=\Delta_{1} q(h, h), w_{2}(h, l)=\Delta_{2} q(h, h)$, and $w_{2}(l, h)=w_{1}(h, h)=w_{2}(h, h)=0$. Using these 
rents, we find that the assembler's expected profit can be written as:

$$
\begin{aligned}
Z_{R}= & \sum_{\mathbf{t}} \beta(\mathbf{t})[S(q(\mathbf{t}))-c(\mathbf{t}) q(\mathbf{t})]-\left[\begin{array}{c}
\beta(l, l)\left(\Delta_{1} q(h, l)+\Delta_{2} q(l, h)\right) \\
+\beta(l, h) \Delta_{1} q(h, h)+\beta(h, l) \Delta_{2} q(h, h)
\end{array}\right] \\
= & \beta(l, l)[S(q(l, l))-c(l, l) q(l, l)] \\
& +\beta(l, h)\left[S(q(l, h))-c(l, h) q(l, h)-\frac{\beta(l, l)}{\beta(l, h)} \Delta_{2} q(l, h)\right] \\
& +\beta(h, l)\left[S(q(h, l))-c(h, l) q(h, l)-\frac{\beta(l, l)}{\beta(h, l)} \Delta_{1} q(h, l)\right] \\
& +\beta(h, h)\left[\begin{array}{l}
S(q(h, h))-c(h, h) q(h, h) \\
-\frac{\beta(l, h)}{\beta(h, h)} \Delta_{1} q(h, h)-\frac{\beta(h, l)}{\beta(h, h)} \Delta_{2} q(h, h)
\end{array}\right] .
\end{aligned}
$$

In the first step in (17), we plug the optimal rents into the first equation in (12). In the second step in (17), we simplify the expression by grouping terms according to the $q(\mathbf{t})$ they involve. This is equivalent to the fourth step in (12). Using the virtual costs shown in equation (15), we observe that equation (17) indeed equals (14).

\subsubsection{Optimal Order Quantities for the Relaxed Problem}

Equation (14) specifies the assembler's expected profit for the relaxed problem, given the order quantities $q(\mathbf{t})$. We next find the order quantities to maximize (14), subject to the feasibility constraint (10). After obtaining these optimal order quantities, we can use equation (11) to determine the corresponding optimal rents. We proceed in two steps. In step 1, we assume that the virtual costs, $v(\mathbf{t})$, as defined in (13), satisfy the following monotonicity condition:

$$
v\left(l, \mathbf{t}_{-i}\right) \leq v\left(h, \mathbf{t}_{-i}\right), \text { for any } \mathbf{t}_{-i} .
$$

As will be seen, if (18) holds, the feasibility constraint (10) is satisfied. Then, in step 2, we consider how to find the optimal order quantities when (18) does not hold.

Step 1: If condition (18) holds, we can characterize the optimal order quantities as follows:

Proposition 1 Suppose that condition (18) holds. For the relaxed problem, the assembler's optimal 
order quantity, denoted by $Q(\mathbf{t})$, satisfies

$$
F(Q(\mathbf{t}))=\left(\frac{r-v(\mathbf{t})}{r}\right)^{+} \text {for any given } \mathbf{t},
$$

where $(z)^{+}=\max (0, z)$, and her optimal profit is $Z_{R}=\sum_{\mathbf{t}} \beta(\mathbf{t})[S(Q(\mathbf{t}))-v(\mathbf{t}) Q(\mathbf{t})]<\bar{Z}$, where $\bar{Z}$ is the full-information profit, as given in (7).

Thus, the optimal order quantities for the assembler can be found by using a newsvendor solution, but with the actual costs, $c(\mathbf{t})$, replaced by the virtual costs, $v(\mathbf{t})$. As discussed in $\mathrm{Hu}$ and Qi (2017), who analyze the optimal BIC mechanism for an assembler, the form of the virtual costs is standard in the economics literature, i.e., the virtual costs defined in (13) include the marginal production cost plus the rent paid to the supplier. Hence, the resulting order quantities are less than the full-information order quantities, as characterized in Section 2.2.

It is now clear that condition (18) guarantees that the optimal order quantities in (19) satisfy the feasibility constraint (10). Since Proposition 1 requires that the virtual costs satisfy condition (18), it is important to identify conditions under which (18) holds, as discussed in the following corollary.

Corollary 1 When the suppliers' types are independently distributed, i.e., $\beta\left(t_{i} \mid \mathbf{t}_{-i}\right)=\beta\left(t_{i}\right)$ for any $t_{i}, i$, and $\mathbf{t}_{-i}$, the virtual costs satisfy condition (18).

In Appendix A, we provide a discussion of our measure of the correlation, denoted by $\rho$, between types in a two-supplier setting. We then use the two-supplier model to show that whenever the cost distribution is non-positively correlated, condition (18), and thus Proposition 1, holds. In Section 4, we discuss the continuous-type model. To ensure that the virtual costs are monotonic, which is the equivalent of (18) in a continuous-type model, we assume that the probability density functions are log-concave, which is commonly assumed in the literature. For example, the multivariate Normal distribution satisfies the log-concavity property (Bagnoli and Bergstorm, 2005).

Step 2: If condition (18) does not hold, a "partial pooling" result is optimal for the order quantities, as we now demonstrate. Recall that we need to find the order quantities to maximize the assembler's profit, as given in (14), while satisfying the feasibility constraint (10). To understand the solution, 
consider the case in which $v\left(l, \mathbf{t}_{-i}\right)>v\left(h, \mathbf{t}_{-i}\right)$ for a particular pair of $\mathbf{t}$ and $i$. In the assembler's profit, the relevant terms, i.e., those involving $q\left(l, \mathbf{t}_{-i}\right)$ and $q\left(h, \mathbf{t}_{-i}\right)$, are as follows:

$$
\beta\left(l, \mathbf{t}_{-i}\right)\left[S\left(q\left(l, \mathbf{t}_{-i}\right)\right)-v\left(l, \mathbf{t}_{-i}\right) q\left(l, \mathbf{t}_{-i}\right)\right]+\beta\left(h, \mathbf{t}_{-i}\right)\left[S\left(q\left(h, \mathbf{t}_{-i}\right)\right)-v\left(h, \mathbf{t}_{-i}\right) q\left(h, \mathbf{t}_{-i}\right)\right] .
$$

This relevant portion of the assembler's profit is concave in both $q\left(l, \mathbf{t}_{-i}\right)$ and $q\left(h, \mathbf{t}_{-i}\right)$. If we ignore the feasibility constraint (10), which requires $q\left(l, \mathbf{t}_{-i}\right) \geq q\left(h, \mathbf{t}_{-i}\right)$, we find that the unconstrained optimal order quantities satisfy $q\left(l, \mathbf{t}_{-i}\right)<q\left(h, \mathbf{t}_{-i}\right)$. This is due to the fact that $(20)$ is separable in $q\left(l, \mathbf{t}_{-i}\right)$ and $q\left(h, \mathbf{t}_{-i}\right)$, which implies that solving for $q\left(l, \mathbf{t}_{-i}\right)$ and $q\left(h, \mathbf{t}_{-i}\right)$ is equivalent to solving two separate newsvendor problems, with objective functions $S\left(q\left(l, \mathbf{t}_{-i}\right)\right)-v\left(l, \mathbf{t}_{-i}\right) q\left(l, \mathbf{t}_{-i}\right)$ and $S\left(q\left(h, \mathbf{t}_{-i}\right)\right)-v\left(h, \mathbf{t}_{-i}\right) q\left(h, \mathbf{t}_{-i}\right)$. Since $v\left(l, \mathbf{t}_{-i}\right)>v\left(h, \mathbf{t}_{-i}\right)$, the optimal quantities satisfy $q\left(l, \mathbf{t}_{-i}\right)<q\left(h, \mathbf{t}_{-i}\right)$. Thus, the concavity of the objective function ensures that the optimal order quantities under the feasibility constraint satisfy $q\left(l, \mathbf{t}_{-i}\right)=q\left(h, \mathbf{t}_{-i}\right)$. We let $q\left(l, \mathbf{t}_{-i}\right)=$ $q\left(h, \mathbf{t}_{-i}\right)=Q\left(\mathbf{t}_{-i}\right)$ and rewrite $(20)$ as:

$$
\begin{aligned}
& \beta\left(l, \mathbf{t}_{-i}\right)\left[S\left(Q\left(\mathbf{t}_{-i}\right)\right)-v\left(l, \mathbf{t}_{-i}\right) Q\left(\mathbf{t}_{-i}\right)\right]+\beta\left(h, \mathbf{t}_{-i}\right)\left[S\left(Q\left(\mathbf{t}_{-i}\right)\right)-v\left(h, \mathbf{t}_{-i}\right) Q\left(\mathbf{t}_{-i}\right)\right] \\
= & {\left[\beta\left(l, \mathbf{t}_{-i}\right)+\beta\left(h, \mathbf{t}_{-i}\right)\right]\left\{S\left(Q\left(\mathbf{t}_{-i}\right)\right)-\frac{\beta\left(l, \mathbf{t}_{-i}\right) v\left(l, \mathbf{t}_{-i}\right)+\beta\left(h, \mathbf{t}_{-i}\right) v\left(h, \mathbf{t}_{-i}\right)}{\beta\left(l, \mathbf{t}_{-i}\right)+\beta\left(h, \mathbf{t}_{-i}\right)} Q\left(\mathbf{t}_{-i}\right)\right\} . }
\end{aligned}
$$

Solving the first-order condition, we find that the optimal pooled order quantity, $Q\left(\mathbf{t}_{-i}\right)$, satisfies

$$
F\left(Q\left(\mathbf{t}_{-i}\right)\right)=\left(\frac{r-v\left(\mathbf{t}_{-i}\right)}{r}\right)^{+}
$$

where $v\left(\mathbf{t}_{-i}\right)$ is called the pooled virtual cost, and is given by

$$
v\left(\mathbf{t}_{-i}\right)=\frac{\beta\left(l, \mathbf{t}_{-i}\right) v\left(l, \mathbf{t}_{-i}\right)+\beta\left(h, \mathbf{t}_{-i}\right) v\left(h, \mathbf{t}_{-i}\right)}{\beta\left(l, \mathbf{t}_{-i}\right)+\beta\left(h, \mathbf{t}_{-i}\right)} .
$$

The pooling solution outlined in Step 2 applies when (18) is violated for a given $\mathbf{t}$ and a single value of $i$. When there are more than two suppliers, it is possible for the condition to be violated for more than one $i$. For example, in a three-supplier case, we may have $v(h, l, h)>$ $v(h, h, h)$ and $v(h, h, l)>v(h, h, h)$. In this case, pooling still occurs, but the solution can be more 
complex. In our example, depending on the specific input parameters, there are multiple possible pooling solutions, including: (i) $q(h, l, h)=q(h, h, l)=q(h, h, h)$; (ii) $q(h, l, h)=q(h, h, h)$ and (iii) $q(h, h, l)=q(h, h, h)$. In each case, analogous to (22) and (23), the pooled quantity is computed based on a weighted average of the virtual costs, while the remaining quantities are set using (19). For settings in which (18) is violated for multiple $i$, the pooled solution can be found by formulating the Lagrangean and considering the complementary slackness conditions, similar to the approach used in Section 2.5 of Vohra (2012). Details regarding how to apply this solution procedure to the assembly setting can be found in Appendix F. Finally, recall that by Corollary 1, when the supplier's costs are independent, (18) holds and pooling is not required.

\subsubsection{Solution Procedure for the Relaxed Problem}

To summarize, we have characterized the optimal order quantities in two cases, i.e., as given by (19) when condition (18) holds and by (22) when condition (18) does not hold. A complete solution procedure requires us to first determine whether or not condition (18) holds, and then proceed accordingly. Thus, the complete solution procedure for the relaxed problem is as follows:

- Apply (13) to determine the virtual costs for all possible vectors of types.

- Determine whether or not the virtual costs satisfy condition (18).

- If condition (18) holds for a given vector of types, apply (19) in Proposition 1 to determine the optimal order quantities.

- If condition (18) does not hold for a given vector of types, determine the appropriate pooling and the pooled virtual cost, and then apply (22) to determine the optimal pooled order quantity.

- After obtaining the order quantities, apply (11) to determine the corresponding markups.

\subsection{Optimal ICDS Mechanism}

We next consider the solution to the assembler's problem, which is to maximize (2) subject to (4) and the IR constraints, i.e., $w_{i}(\mathbf{t}) \geq 0$. We start by checking whether the solution to the relaxed problem, as characterized in Section 2.3, satisfies the omitted constraints (9). Using the optimal 
rents for the relaxed problem, as given in (11), we find that the omitted constraints (9) become:

$$
w_{i}\left(h, \mathbf{t}_{-i}\right)=0 \geq \Delta_{i}\left[q\left(h, \mathbf{t}_{-i}\right)-q_{i}\left(l, \mathbf{t}_{-i}\right)\right],
$$

which holds whenever the feasibility constraint (10) is satisfied. Since the solution to the relaxed problem, as characterized in Section 2.3, satisfies (10), we conclude that the relaxed solution solves the fully constrained model. Formally, we have the following result:

Proposition 2 The optimal solution for the relaxed problem solves the assembler's fully constrained problem, i.e., maximize (2) subject to (4) and $w_{i}(\mathbf{t}) \geq 0$. Therefore, the optimal ICDS mechanism always exists and can be found using the steps described in Section 2.3.3. In addition, the optimal procurement quantities are balanced, i.e., they satisfy $q_{1}(\mathbf{t})=q_{2}(\mathbf{t})=\ldots=q_{n}(\mathbf{t})$.

We can now apply the envelope theorem to understand how the input parameters affect the assembler's optimal profit. Equation (14) indicates that the assembler's optimal profit is decreasing with respect to the virtual cost. Since an increase in $c_{i}(h)$ results in a larger value of $v(\mathbf{t})$, we know that the assembler's optimal profit decreases with $c_{i}(h)$. However, the change in the assembler's profit is indefinite with respect to $c_{i}(l)$, with $c_{i}(h)$ fixed. An increase in $c_{i}(l)$, with all else unchanged, would increase the first term of $v(\mathbf{t})$ in equation (13). However, $\Delta_{i}=c_{i}(h)-c_{i}(l)$ would decrease. A reduced $\Delta_{i}$ could lower the second term of $v(\mathbf{t})$ in equation (13).

Finally, note that the optimal ICDS mechanism does not require the suppliers to know the joint probability distribution of the types, $\beta(\mathbf{t})$. The assembler is the only player who must possess knowledge of $\beta(\mathbf{t})$. From an implementation perspective, this observation is critical given that the suppliers may be geographically dispersed and have little knowledge of each other's cost structure.

\subsection{A Hybrid Mechanism}

While the ICDS mechanism, as characterized above, outperforms the FRW mechanism, it is also more complex to implement. Therefore, to reduce the computational effort and complexity of the contracts, we propose an easier to implement hybrid mechanism.

The idea behind the hybrid mechanism is that assemblers often contract with a very large number of suppliers. However, not all suppliers (or components) are of equal importance to, or have 
equal impact on, the assembler. Since we assume that the final product requires a single unit of each component, volume is not a determinant of the importance of a given supplier. Therefore, it makes sense to measure the importance of a given supplier by $\Delta_{i}$, which represents the degree of uncertainty the assembler has regarding supplier $i$ 's $\operatorname{cost}^{1}$. The ICDS mechanism is designed to maximize the assembler's profit when there is uncertainty regarding the suppliers' costs. However, for suppliers with low uncertainty, using the optimal mechanism may be of marginal value, while creating additional complexity related to implementation. Thus, the proposed hybrid mechanism uses the sophisticated ICDS mechanism for suppliers with high uncertainty (large $\Delta_{i}$ ) and the simpler naïve mechanism (see Definition 1), for suppliers with low uncertainty (small $\Delta_{i}$ ). Specifically, our proposed mechanism works as follows:

- First, the assembler sorts the suppliers (components) such that $\Delta_{i}$ is decreasing with $i$. In other words, the suppliers should be indexed so that $\Delta_{1} \geq \Delta_{2} \geq \cdots \geq \Delta_{n}$.

- Second, the assembler chooses an integer $k \in\{0,1,2, \ldots, n\}$ to determine the complexity of the hybrid mechanism. As will be seen, a larger value of $k$ implies a higher level of complexity and higher profit for the assembler. The assembler invites the first $k$ suppliers, i.e., supplier $j$ for $j \in\{1, \ldots, k\}$, as indexed in the first step, to participate in the ICDS mechanism.

- Third, the assembler contacts the remaining $n-k$ suppliers and offers a wholesale price contract to each of them. The wholesale price contract specifies that the assembler will pay $c_{j}(h)$ for each unit of component $j$ procured from supplier $j$, for $j \in\{k+1, \ldots, n\}$. This ensures that all suppliers accept the offered contract and earn non-negative surplus. If the assembler offered these suppliers a contract in which they were paid an amount less than $c_{j}(h)$, some suppliers (i.e., those who are truly high-cost) would find the contract unprofitable, and thus they would choose not to participate in the contract. Since the assembler requires all components to make the final product, he must design a mechanism in which all suppliers accept the offered contracts. The contract offer to the remaining $n-k$ suppliers does not specify the quantity to be procured until after the first $k$ suppliers have reported their types.

- Fourth, the first $k$ suppliers participate in the ICDS mechanism and independently report their types. The assembler determines the payments and order quantity, $q\left(t_{1}, t_{2}, \ldots, t_{k}\right)$, for these $k$ suppliers. The assembler also orders $q\left(t_{1}, t_{2}, \ldots, t_{k}\right)$ from the remaining $n-k$ suppliers.

The order quantity under the hybrid mechanism is still balanced. However, it now depends on only $k$ suppliers' reported types. For any given $k$, to find the optimal hybrid mechanism, we simply

\footnotetext{
${ }^{1}$ As can be seen in equation (11), the rent paid to each supplier is increasing with that supplier's $\Delta_{i}$. In addition, as discussed above, the assembler's profit decreases as $c_{i}(h)$ increases, with all else fixed, which implies that, for a given $c_{i}(l)$, the assembler's profit decreases as $\Delta_{i}=c_{i}(h)-c_{i}(l)$ increases.
} 
add the following constraints to the ICDS model described in the previous section:

$$
q\left(t_{1}, t_{2}, \ldots, t_{k}, t_{k+1}, \ldots, t_{n}\right)=q\left(t_{1}, t_{2}, \ldots, t_{k}\right) \quad \text { for any }\left(t_{k+1}, \ldots, t_{n}\right) \text { and }\left(t_{1}, t_{2}, \ldots, t_{k}\right)
$$

Constraints (25) ensure that the order quantity depends only on the reported types of the first $k$ suppliers. Therefore, under the hybrid mechanism, the assembler wishes to maximize the expected profit in equation (14), subject to the hybrid constraints (25), the downward IC constraints (8), and the feasibility constraints (5). See Appendix E for the complete formulation of the hybrid problem. Constraints (25) create additional "partial pooling" in the ICDS solution.

To compute the hybrid mechanism, we first use the procedure described in Section 2.3 to determine the virtual costs, $v\left(t_{1}, t_{2}, \ldots, t_{n}\right)$, for the original ICDS model. Note that, due to the feasibility constraints, (5), this may involve some partial pooling. Then, we use $v\left(t_{1}, t_{2}, \ldots, t_{n}\right)$ as the inputs to equation (26) below to determine the hybrid virtual costs, denoted by $\tilde{v}\left(t_{1}, t_{2}, \ldots, t_{k}\right)$ :

$$
\tilde{v}\left(t_{1}, t_{2}, \ldots, t_{k}\right)=E\left[v\left(t_{1}, t_{2}, \ldots, t_{k}, t_{k+1}, \ldots, t_{n} \mid t_{1}, t_{2}, \ldots, t_{k}\right)\right]
$$

where the expectation is taken with respect to $\left(t_{k+1}, \ldots, t_{n}\right)$, i.e., with respect to the types of the $n-k$ suppliers not included in the ICDS mechanism. We then have the following result:

Proposition 3 For a given $k \in\{0,1,2, \ldots, n\}$, the optimal hybrid mechanism has the following properties:

1) The balanced order quantity $\tilde{q}\left(t_{1}, t_{2}, \ldots, t_{k}\right)$ satisfies

$$
F\left(\tilde{q}\left(t_{1}, t_{2}, \ldots, t_{k}\right)\right)=\left(\frac{r-\tilde{v}\left(t_{1}, t_{2}, \ldots, t_{k}\right)}{r}\right)^{+}
$$

2) The rent for supplier $i \in\{1,2, \ldots, k\}$ is

$$
w_{i}\left(l, \tilde{\mathbf{t}}_{-i}\right)=\Delta_{i} q\left(h, \tilde{\mathbf{t}}_{-i}\right) \text { and } w_{i}\left(h, \tilde{\mathbf{t}}_{-i}\right)=0 \text {, for any } \tilde{\mathbf{t}}_{-i},
$$

where the type vector is $\tilde{\mathbf{t}}=\left(t_{1}, t_{2}, \ldots, t_{k}\right)$, which has $k$ elements. 
3) The payment to the remaining $n-k$ suppliers equals

$$
P_{i}\left(t_{1}, t_{2}, \ldots, t_{k}\right)=c_{i}(h) \tilde{q}\left(t_{1}, t_{2}, \ldots, t_{k}\right) \quad \text { for } i \in\{k+1, \ldots, n\} .
$$

4) The assembler's expected profit equals $\tilde{Z}=\sum_{\tilde{\mathbf{t}}} \beta(\tilde{\mathbf{t}})[S(\tilde{q}(\tilde{\mathbf{t}}))-\tilde{v}(\mathbf{t}) \tilde{q}(\tilde{\mathbf{t}})]$.

For example, consider a case with $n=3$ and $k=2$, where $\Delta_{1}>\Delta_{2}>\Delta_{3}$. The assembler designs an ICDS mechanism for suppliers 1 and 2, and offers supplier 3 a wholesale price contract with payment of $c_{3}(h)$ per unit. The ICDS mechanism has $\tilde{q}\left(t_{1}, t_{2}\right)=\tilde{q}\left(t_{1}, t_{2}, h\right)=\tilde{q}\left(t_{1}, t_{2}, l\right)$, which can be computed from (27) using

$$
\tilde{v}\left(t_{1}, t_{2}\right)=\frac{\beta\left(t_{1}, t_{2}, l\right)}{\beta\left(t_{1}, t_{2}, l\right)+\beta\left(t_{1}, t_{2}, h\right)} v\left(t_{1}, t_{2}, l\right)+\frac{\beta\left(t_{1}, t_{2}, h\right)}{\beta\left(t_{1}, t_{2}, l\right)+\beta\left(t_{1}, t_{2}, h\right)} v\left(t_{1}, t_{2}, h\right),
$$

where $v\left(t_{1}, t_{2}, l\right)$ and $v\left(t_{1}, t_{2}, h\right)$ are found using the procedure described in Section 2.3 for the ICDS mechanism with $n=2$.

There are two important special cases for the hybrid mechanism. When $k=0$, there is only one hybrid virtual cost, which is $\tilde{v}\left(t_{1}, t_{2}, \ldots, t_{n}\right)=E\left[v\left(t_{1}, t_{2}, \ldots, t_{n}\right)\right]=c(\mathbf{h})=\sum_{i=1}^{n} c_{i}(h)$. Thus, the order quantity is independent of the suppliers' types. Notice that this special case reduces to the naïve mechanism, as defined in Section 2.2, in which the assembler assumes that all suppliers are high-cost. The second special case is when $k=n$. In this case, the hybrid mechanism is the same as the ICDS mechanism because $\tilde{v}\left(t_{1}, t_{2}, \ldots, t_{n}\right)=E\left[v\left(t_{1}, t_{2}, \ldots, t_{n} \mid t_{1}, t_{2}, \ldots, t_{n}\right)\right]=v\left(t_{1}, t_{2}, \ldots, t_{n}\right)$.

In summary, the hybrid mechanism is easier to implement than the ICDS mechanism when $k<n$. In addition, both the complexity of the hybrid mechanism and the assembler's expected profit are increasing in $k$. Therefore, when applying the hybrid mechanism and choosing the best $k$, the assembler must trade-off the benefits of reduced complexity with the loss of profit associated with a lower $k$. In Section 3.2, we present a comprehensive numerical study to evaluate the performance of the hybrid mechanism and demonstrate this trade-off. 


\section{Numerical Study}

In this section, we present the results of a comprehensive numerical study which we use to demonstrate that the ICDS mechanism can significantly outperform the FRW mechanism. We also demonstrate that the proposed hybrid mechanism can outperform the FRW mechanism and, in some cases, achieves an expected profit for the assembler that is close to the optimal expected profit under the ICDS mechanism. In Section 3.1, to compare the ICDS and FRW mechanisms, we consider a setting with $n=2$. In Section 3.2, we consider more realistic problem instances with $n \in\{4,6,8,10\}$ suppliers in order to also evaluate the performance of the hybrid mechanism.

\subsection{Performance of ICDS and FRW Mechanisms for $n=2$ Suppliers}

For a setting with $n=2$, we created a set of $2,400(=5 \times 6 \times 4 \times 4 \times 5)$ test problems, as follows:

- The high-cost suppliers' unit production costs: $c_{1}(h) \in\{5,7,9,11,13\}$ and $c_{2}(h) \in\{2,3,4,5,6,7\}$.

- The low-cost suppliers' unit production costs are assigned fixed values: $c_{1}(l)=3$ and $c_{2}(l)=1$.

- The probability that the supplier is high-cost: $p_{1}(h) \in\{0.2,0.4,0.6,0.8\}$ and $p_{2}(h) \in\{0.2,0.4,0.6,0.8\}$. We assumed that $\beta\left(t_{1}, t_{2}\right)=p_{1}\left(t_{1}\right) p_{2}\left(t_{2}\right)$, i.e., the suppliers' types are independent.

- Demand is uniformly distributed on the interval [500- $\alpha, 500+\alpha]$, for $\alpha \in\{100,200,300,400,500\}$, where $\alpha$ is a measure of the level of uncertainty in demand. Higher $\alpha$ implies more uncertainty.

- In all of the experiments, the selling price $r$ is fixed at 30 .

Since the feasible region for the ICDS mechanism is less constrained than for the FRW mechanism, the optimal ICDS mechanism provides the assembler with higher profit than the optimal FRW mechanism. Across the 2,400 test problems, the average percentage increase in profit under the ICDS mechanism, relative to the FRW mechanism, was $10.38 \%$, with a standard deviation of $13.78 \%$. Thus, the numerical results indicate that the increase in profit under the ICDS mechanism can be substantial. Figure 1 demonstrates the performance of the ICDS mechanism, relative to the FRW mechanism, for various problem parameters. We summarize the key results below:

- The gain from the ICDS mechanism is increasing in $\Delta_{i}=c_{i}(h)-c_{i}(l)$, for $i \in\{1,2\}$. Thus, when there is more uncertainty regarding the suppliers' unit costs, it is particularly important for the assembler to use the ICDS mechanism, rather than the simpler FRW mechanism.

- The gain from the ICDS mechanism is increasing with the level of uncertainty in demand, $\alpha$. This is due to the fact that the ICDS mechanism allows the buyer to make quantity decisions 


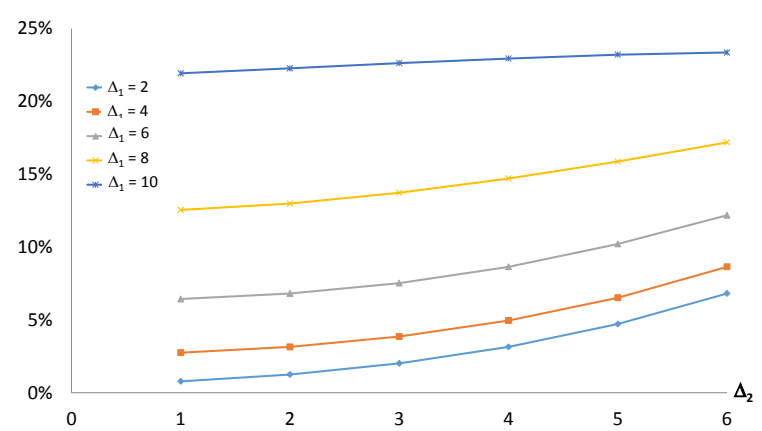

(a) Impact of $\Delta_{1}$ and $\Delta_{2}$, for $p_{1}(h)=0.4, p_{2}(h)=0.4$

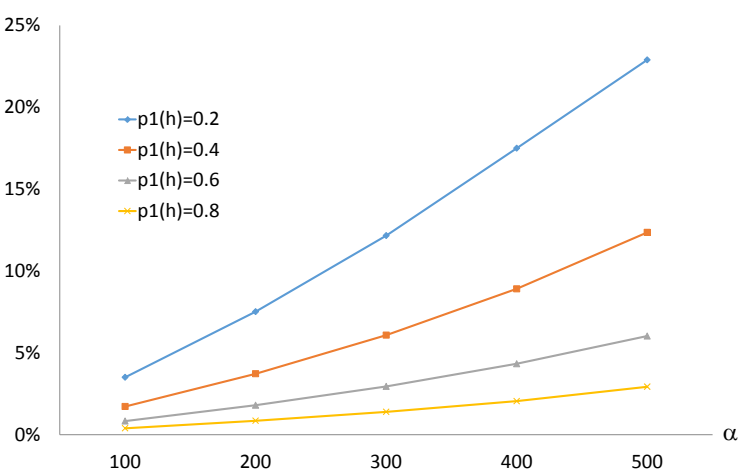

(b) Impact of $p_{1}(h)$ and $\alpha$, for $c_{1}(h)=9, c_{2}(h)=2$

Figure 1: Percentage Gain in Assembler's Expected Profit under the ICDS Mechanism, Relative to the FRW Mechanism

using information on all of the suppliers' costs, while the FRW mechanism does not. Thus, coordination of the quantity decision becomes more important when the demand uncertainty increases, i.e., when the uncertainty in demand is high, setting the order quantity properly, by incorporating information regarding all suppliers' types, becomes critical.

- The gain from the ICDS mechanism is decreasing in the marginal probability that a supplier is the high-cost type, $p_{1}(h)$. Thus, when there is a small probability that one of the suppliers is high-cost, it is important for the assembler to use the ICDS mechanism. Recall that a high-cost supplier does not receive information rent under either the ICDS or the FRW mechanisms. Thus, as the probability that a supplier is high-cost increases, the performance of the two mechanisms becomes more similar.

\subsection{Performance of ICDS, Hybrid, and FRW Mechanisms}

In practice, assemblers often need to contract with a large number of distinct suppliers. Therefore, we conducted an additional set of experiments with $n \in\{4,6,8,10\}$ suppliers. We are interested in assessing the performance of the hybrid mechanism and the ICDS mechanism, relative to the FRW mechanism. To do so, we consider 800 problem instances using the following parameter values:

- Fixed $c(l)$ problem instances: We fix the low cost across the set of suppliers, i.e., we set $c_{i}(l)=c(l)$ for $i=1, \ldots, n$, but we allow the high cost, $c_{i}(h)$, to vary across the suppliers. We consider four different values of $c(l)$, i.e., $c(l) \in\{1,6,11,16\}$. For a given $c(l)$, we set $c_{i}(h)=30-i$ for supplier $i=1, \ldots, n$.

- Fixed $c(h)$ problem instances: We fix the high cost across the set of suppliers, i.e., we set $c_{i}(h)=c(h)$ for $i=1, \ldots, n$, but we allow the low cost, $c_{i}(l)$, to vary across the suppliers. We consider four different values of $c(h)$, i.e., $c(h) \in\{11,16,21,26\}$. For a given $c(h)$, we set $c_{i}(l)=i$ for supplier $i=1, \ldots, n$. 
- In each set of problem instances, the marginal probability distribution for the suppliers' unit costs is set to be the same for all 10 suppliers, i.e., we set $p_{i}(h)=p(h)$ for $i=1, \ldots, n$, and we consider four different values, i.e., we set $p(h) \in\{0.2,0.4,0.6,0.8\}$. We assume that each supplier's type is independent of the other suppliers' types.

- In each set of problem instances, the range for the uniform distribution of demand is [1000 $\alpha, 1000+\alpha]$ for $\alpha \in\{200,400,600,800,1000\}$.

- In each set of problem instances, the selling price for the end-product is $r \in\{270,280,290,300,310\}$. These values ensure that $c(\mathbf{h})=\sum_{i=1}^{n} c_{i}(h)<r$ in all experiments.

For a given $n$, we have $400(=4 \times 4 \times 5 \times 5)$ problem instances with a fixed $c(l)$ and another $400(=4 \times 4 \times 5 \times 5)$ problem instances with a fixed $c(h)$. For each, we solve for the optimal FRW, hybrid, and ICDS mechanism. Thus, we solve a total of 2400 problems for each $n \in\{4,6,8,10\}$.

Recall that $\Delta_{i}=c_{i}(h)-c_{i}(l)$ measures the degree of uncertainty regarding supplier $i$ 's costs. In the fixed $c(l)$ and $c(h)$ problem instances, we have $\Delta_{i}=30-c(l)-i$ and $\Delta_{i}=c(h)-i$, respectively, for $i=1, \ldots, n$. Thus, for the fixed $c(l)$ and $c(h)$ problem instances, $\Delta_{i}$ is decreasing in $c(l)$ and increasing in $c(h)$, respectively. Finally, also recall that the hybrid mechanism selects the $k$ suppliers with the largest $\Delta_{i}$ for contracting under the ICDS mechanism.

The experimental results, for the fixed $c(l)$ and fixed $c(h)$ instances, for $n=10$, are shown in Tables 3 and 4, respectively, in Appendix C. The tables show the percentage gain (i.e., percentage increase in expected profits for the assembler) from using the hybrid (with $k=3$ ) and ICDS mechanisms, relative to the FRW mechanism. On average, the profit from the hybrid (ICDS) mechanism is $29.5 \%(67.3 \%)$ higher than the profit from the FRW mechanism for the fixed $c(l)$ problem instances, and $38.8 \%(90.6 \%)$ higher for the fixed $c(h)$ problem instances. Table 5 in Appendix D presents additional experimental results to further demonstrate this point.

As shown in Tables 3 and 4, the impact of the key input parameters is consistent with the results provided in Section 3.1 for $n=2$. In particular, the value of the more sophisticated hybrid and ICDS mechanisms, compared to the FRW mechanism, is increasing in the uncertainty regarding demand $(\alpha)$, the probability that the supplier is low-cost $(p(l)=1-p(h))$, and the uncertainty regarding the suppliers' costs (smaller $c(l)$ and larger $c(h)$ ). In addition, the more sophisticated mechanisms add more value when the profit margin, i.e., the unit selling price $(r)$, is small.

We can also use these experiments to compare the performance of the hybrid and ICDS mechanisms. For the fixed $c(l)$ (fixed $c(h)$ ) problems with $n=10$, the ICDS mechanism provides, on 


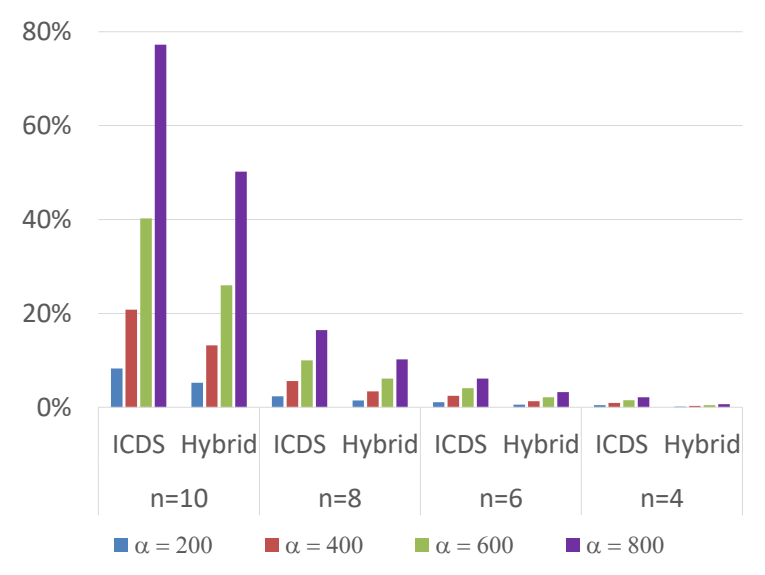

(a) Impact of $n$ and $\alpha$ for fixed $c(h)$ instances

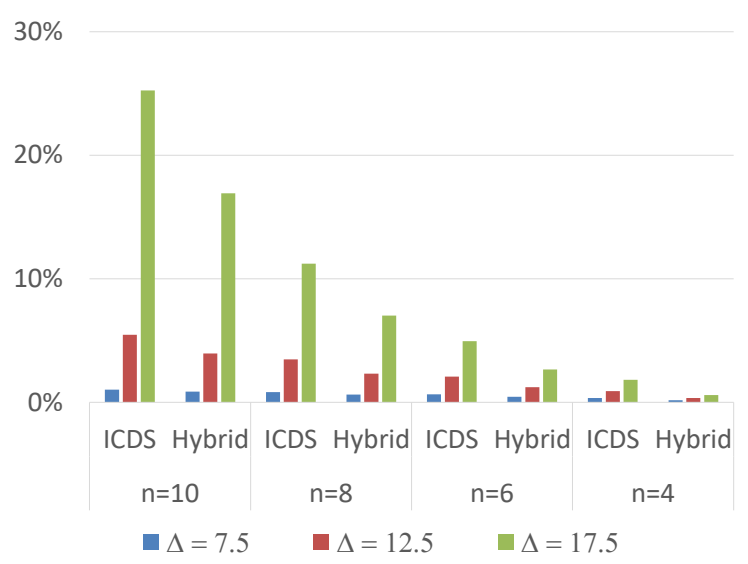

(b) Impact of $n$ and average $\Delta_{i}$ for fixed $c(h)$ instances

Figure 2: Percentage Gain from Hybrid and ICDS Mechanisms Relative to FRW Mechanism average, a 21\% (14\%) gain relative to the hybrid mechanism with $k=3$. Therefore, using the ICDS mechanism, rather than the hybrid mechanism, is more important for the fixed $c(l)$ problems than for the fixed $c(h)$ problems. For $n=10$, the average $\Delta_{i}=c_{i}(h)-c_{i}(l)$ is 16.5 (13) for the fixed $c(l)$ (fixed $c(h))$ problems. Thus, the value of the ICDS mechanism, relative to the hybrid mechanism, is greatest when there is more uncertainty regarding the suppliers' unit costs (i.e., larger $\Delta_{i}$ ). Further, using the ICDS mechanism is most critical when demand uncertainty is high (large $\alpha$ ), the assembler's expected profit margin is low (small $r$ ), and the probability a supplier is high-cost is low (small $p(h))$. For the last point, recall that the naïve mechanism used as part of the hybrid mechanism assumes the suppliers have high cost. Thus, the hybrid mechanism performs worse when that assumption is less likely to be correct.

\subsubsection{Impact of Number of Suppliers on ICDS, Hybrid, and FRW Mechanisms}

Next, we study the impact of the number of suppliers on the relative performance of the ICDS, hybrid and FRW mechanisms. We consider $n \in\{4,6,8,10\}$ and, for the hybrid mechanism, we set $k=n / 2$. The percentage gains from using the ICDS and hybrid mechanisms, relative to the FRW mechanism, are shown in Figure 2, for the fixed $c(h)$ problem instances ${ }^{2}$.

Figure 2(a) shows the impact of the uncertainty in demand $(\alpha)$, while Figure 2(b) shows the impact of the uncertainty regarding the suppliers' costs (average value of $\Delta_{i}$ across the suppliers).

\footnotetext{
${ }^{2}$ Figure 2 does not show the cases with $\alpha=1000$ and $c(h)=26$ (average $\Delta_{i}=22.5$ ). The percentage gains for these cases are very large (between $193 \%$ and $331 \%$ ) and require an axis scale that makes the results difficult to see.
} 
The figures demonstrate that the percentage gains from using the more sophisticated ICDS and hybrid mechanisms, relative to the FRW mechanism, increase significantly as the number of suppliers increases, with the greatest gains when there is high uncertainty regarding demand and high uncertainty regarding the suppliers' costs. As discussed in Section 1, many assemblers (e.g., those in the automotive industry) operate in environments with a very large number of suppliers and a high degree of uncertainty in both demand and their suppliers' costs. The results in Figure 2 suggest that by using the hybrid or ICDS mechanisms, rather than the simpler FRW mechanism, the assembler can significantly improve her profit.

We next explain the poor performance of the FRW mechanism relative to the hybrid and ICDS mechanisms when $n$ becomes large. In all of our numerical experiments with $n=10$, we find that the FRW mechanism (as determined using Algorithm 1 in Fang et al. (2014)) reduces to the naïve mechanism. Further investigation reveals that, when there is a large number of suppliers or when the suppliers have similar cost structure, Step 2 of Algorithm 1 in Fang et al. (2014) causes the optimal order quantities for the suppliers to merge into a single order quantity. When this happens, Theorem 2 in Fang et al. (2014) implies that the optimal solution of the FRW mechanism is identical to that of the naïve mechanism. Therefore, for all of the parameter settings used in our experiments with $n=10$, the FRW mechanism reduces to naïve mechanism, in which the assembler assumes that all suppliers are high-cost. Hence, for all of these settings, the FRW mechanism cannot be used by the assembler to eliminate any of the inefficiency caused by information asymmetry. Thus, for practical settings with a moderate to large number of suppliers, a more sophisticated mechanism, such as the hybrid or ICDS mechanisms, is required for the assembler to effectively cope with asymmetric information regarding the suppliers' costs.

\subsubsection{Impact of $k$ on Performance of Hybrid Mechanism}

The hybrid mechanism requires the assembler to determine $k$, i.e., the number of suppliers to contract with under an ICDS mechanism. As noted above, both the complexity of the hybrid mechanism, and the assembler's expected profit, are increasing in $k$. To provide insights that can be used by an assembler to determine the appropriate value of $k$, we present results for the experiments described in Section 3.2 for the case with $n=10$. In these experiments, we vary the 


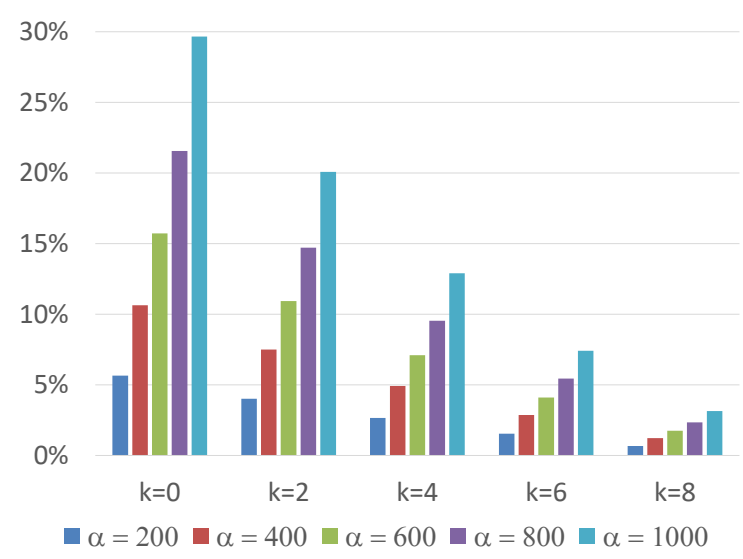

(a) Impact of $k$ and $\alpha$ for fixed $c(h)$ instances

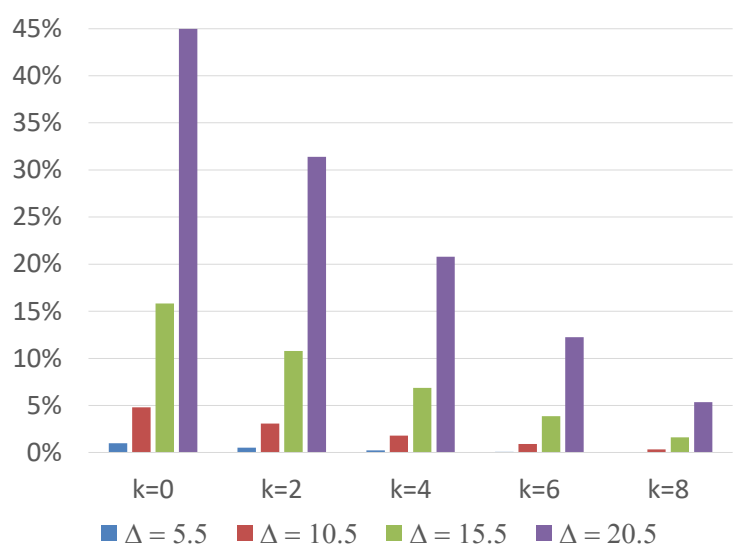

(b) Impact of $k$ and average $\Delta_{i}$ for fixed $c(h)$ instances

Figure 3: Percentage Loss from Hybrid Mechanism Relative to ICDS Mechanism

value of $k$.

Figure 3 shows the percentage loss under the hybrid mechanism, relative to the ICDS mechanism, for the fixed $c(h)$ problem instances. Figure 3(a) considers the impact of $k$ and the level of demand uncertainty, measured by $\alpha$, while Figure 3(b) considers the impact of $k$ and the level of uncertainty regarding the suppliers' costs, as measured by the average value of $\Delta_{i}$ across the $n=10$ suppliers. The figures indicate that the loss from using the hybrid mechanism decreases as $k$ increases, and increases in both $\alpha$ and the average $\Delta_{i}$. These results imply that using a large value of $k$ is particularly important for assemblers that face high demand uncertainty and significant uncertainty regarding their suppliers' costs.

\section{Extensions to Continuous-type Model}

Finally, we consider the continuous version of the model, which is identical to the model considered in Section 2, except that we now assume that the suppliers' costs (or types) follow a continuous joint distribution. Specifically, we use a non-negative random variable, denoted by $x_{i}$, to represent the cost for supplier $i$ to produce one unit of component $i$. Let $\mathbf{x}=\left(x_{1}, x_{2}, \ldots, x_{n}\right)$ denote the vector of costs and let $\mathbf{x}_{-i}=\left(x_{1}, \ldots, x_{i-1}, x_{i+1}, \ldots, x_{n}\right)$ denote the vector of costs excluding $x_{i}$. The joint probability density function of $\mathbf{x}$ is given by $g(\mathbf{x})=g_{i}\left(x_{i} \mid \mathbf{x}_{-i}\right) g_{-i}\left(\mathbf{x}_{-i}\right)$, where $g_{i}\left(x_{i} \mid \mathbf{x}_{-i}\right)$ is the conditional density function of $x_{i}$, and $g_{-i}\left(\mathbf{x}_{-i}\right)$ is the joint probability density function 
of $\mathbf{x}_{-i}$. In the special case where the costs are independent, $g_{i}\left(x_{i} \mid \mathbf{x}_{-i}\right)=g_{i}\left(x_{i}\right)$ and $g(\mathbf{x})=$ $g_{1}\left(x_{1}\right) \times g_{2}\left(x_{2}\right) \times \ldots \times g\left(x_{n}\right)$. Similarly, we define the associated cumulative distribution functions $G(\mathbf{x}), G_{i}\left(x_{i} \mid \mathbf{x}_{-i}\right)$, and $G_{-i}\left(\mathbf{x}_{-i}\right)$. We also assume that each supplier's per unit cost has a finite upper bound, i.e., $x_{i} \leq \bar{x}_{i}<\infty$, and that $r>\sum_{i=1}^{n} \bar{x}_{i}$, which implies that the naïve mechanism allows the assembler to earn a positive expected profit.

Each supplier has private information regarding their costs, while the assembler only knows the distribution of the costs. The assembler commits to a mechanism and asks each supplier to independently send a message, $m_{i}$, regarding their cost, $x_{i}$. The mechanism is a mapping from the vector of the suppliers' reported costs, $\mathbf{m}=\left(m_{1}, m_{2}, \ldots, m_{n}\right)$, to the procurement quantity vector, $\mathbf{q}(\mathbf{m})=$ $\left(q_{1}(\mathbf{m}), q_{2}(\mathbf{m}), \ldots, q_{n}(\mathbf{m})\right)$, and the payment vector, $\mathbf{P}(\mathbf{m})=\left(P_{1}(\mathbf{m}), P_{2}(\mathbf{m}), \ldots, P_{n}(\mathbf{m})\right)$. Following the standard mechanism design literature (Myerson 1979), we focus on the truth-revealing equilibrium where the reported costs are the same as the suppliers' true costs, i.e, $\mathbf{x}=\mathbf{m}$.

The assembler's objective is to maximize her expected profit, while ensuring that each supplier reports their true costs. We formulate the assembler's problem as follows

$$
\max \quad Z=E_{\mathbf{x}}\left[S(\min (\mathbf{q}(\mathbf{x})))-\sum_{i=1}^{n} P_{i}(\mathbf{x})\right]
$$

subject to

$$
\begin{aligned}
& P_{i}\left(x_{i}, \mathbf{x}_{-i}\right)-x_{i} q_{i}\left(x_{i}, \mathbf{x}_{-i}\right) \geq P_{i}\left(m_{i}, \mathbf{x}_{-i}\right)-x_{i} q_{i}\left(m_{i}, \mathbf{x}_{-i}\right), \quad \forall m_{i} \neq x_{i}, i, x_{i}, \mathbf{x}_{-i} \\
& P_{i}\left(x_{i}, \mathbf{x}_{-i}\right)-x_{i} q_{i}\left(x_{i}, \mathbf{x}_{-i}\right) \geq 0, \quad \forall i, x_{i}, \mathbf{x}_{-i} .
\end{aligned}
$$

The expectation in (28) is with respect to the cost vector $\mathbf{x}$ and $\min (\mathbf{q}(\mathbf{x}))=\min _{i}\left\{q_{i}(\mathbf{x})\right\}$ is the minimum of the procurement quantities from all suppliers. We refer to the constraints in (29) as the IC constraints in dominant strategies for the continuous model, which guarantee that truth-revealing is a dominating strategy for each supplier. The constraints in (30) are the individual rationality (IR) constraints, which ensure that each supplier earns a non-negative profit in equilibrium.

To solve the above mechanism design problem, we start with the definition of the virtual cost 
for our continuous model, which for supplier $i$ can be written as

$$
J_{i}\left(x_{i} \mid \mathbf{x}_{-i}\right)=x_{i}+\frac{G_{i}\left(x_{i} \mid \mathbf{x}_{-i}\right)}{g_{i}\left(x_{i} \mid \mathbf{x}_{-i}\right)}
$$

It is interesting to note that (31) extends the standard virtual cost definition in multi-agent mechanism design to a setting in which the suppliers' costs are not necessarily independent. If the suppliers' costs are independent, the virtual cost becomes $J_{i}\left(x_{i} \mid \mathbf{x}_{-i}\right)=x_{i}+G_{i}\left(x_{i}\right) / g_{i}\left(x_{i}\right)=J_{i}\left(x_{i}\right)$, which is identical to the virtual cost obtained in a single-supplier model, e.g., Theorem 1 of Chaturvedi and Martínez-de-Albéniz (2011). If the distribution of the suppliers' costs satisfies the log-concave property, the virtual cost $J_{i}\left(x_{i} \mid \mathbf{x}_{-i}\right)$ is non-decreasing with respect to $x_{i}$. Logconcave distributions include many well-known distributions, such as the Uniform, Normal, and Weibull, that are commonly used in the mechanism design literature (Bagnoli and Bergstrom 2005).

Following a standard approach, we first establish the properties of feasible mechanisms. We then characterize the optimal mechanism for the assembler. We start by presenting Lemma 3, which provides a set of conditions that are equivalent to the IC and IR constraints, (29) and (30).

Lemma 3 The payment function, $\mathbf{P}(\mathbf{m})=\left(P_{1}(\mathbf{m}), P_{2}(\mathbf{m}), \ldots, P_{n}(\mathbf{m})\right)$, and the ordering policy, $\mathbf{q}(\mathbf{m})=\left(q_{1}(\mathbf{m}), q_{2}(\mathbf{m}), \ldots, q_{n}(\mathbf{m})\right)$, are feasible if and only if, for all $i, x_{i}$, and $\mathbf{x}_{-i}$, the following conditions hold:

$$
\begin{aligned}
\frac{\partial q_{i}\left(x_{i}, \mathbf{x}_{-i}\right)}{\partial x_{i}} & \leq 0 \\
\frac{\partial P_{i}\left(x_{i}, \mathbf{x}_{-i}\right)}{\partial x_{i}}-x_{i} \frac{\partial q_{i}\left(x_{i}, \mathbf{x}_{-i}\right)}{\partial x_{i}} & =0 \\
P_{i}\left(x_{i}, \mathbf{x}_{-i}\right)-x_{i} q_{i}\left(x_{i}, \mathbf{x}_{-i}\right) & \geq 0 .
\end{aligned}
$$

Condition (32) indicates that, in a feasible mechanism, the order quantity from each supplier is nonincreasing with respect to the unit cost. This condition is referred to as the monotonicity constraint in the mechanism design literature. Condition (33) is essentially the first order condition for each supplier's optimization problem, and is referred to as the local IC constraint. Condition (34) gives the IR constraints. Lemma 3 implies that these conditions together ensure that truth-revealing is 
the best strategy for each supplier, and thus they are equivalent to the IC constraints in the original mechanism design formulation. We are now ready to solve the assembler's optimization problem. The following proposition characterizes the optimal procurement mechanism.

Proposition 4 Suppose the virtual costs, $J_{i}\left(x_{i} \mid \mathbf{x}_{-i}\right)$, are non-decreasing with respect to $x_{i}$. In the continuous model, the assembler's optimal mechanism exhibits the following properties:

1) The order quantities are balanced, i.e., $q_{1}(\mathbf{x})=q_{2}(\mathbf{x})=\ldots=q_{n}(\mathbf{x})=Q(\mathbf{x})$, which satisfies

$$
F(Q(\mathbf{x}))=\left(\frac{r-\sum_{i=1}^{n} J_{i}\left(x_{i} \mid \mathbf{x}_{-i}\right)}{r}\right)^{+} .
$$

2) The payment to supplier $i$ equals

$$
P_{i}\left(x_{i}, \mathbf{x}_{-i}\right)=x_{i} Q(\mathbf{x})+\int_{x_{i}}^{\bar{x}_{i}} Q\left(x, \mathbf{x}_{-i}\right) d x .
$$

3) The assembler's expected profit equals

$$
Z^{*}=E_{\mathbf{x}}\left[S(Q(\mathbf{x}))-\left(\sum_{i=1}^{n} J_{i}\left(x_{i} \mid \mathbf{x}_{-i}\right)\right) Q(\mathbf{x})\right]
$$

Proposition 4 shows that the optimal mechanism for the continuous model resembles that for the discrete model. As in the discrete case, the optimal order quantity in (35) is a newsvendor quantity, but with the actual costs replaced by the virtual costs. Since the virtual cost is non-decreasing with respect to $x_{i}$, we have that the order quantity is decreasing in supplier $i$ 's unit cost. The payment to supplier $i$ is given in (36), where the second term represents the markup paid to supplier $i$. This expression indicates that the markup is decreasing with respect to $x_{i}$. Thus, a supplier with a low unit cost receives a larger information rent than does a supplier with a high unit cost.

\section{Conclusions and Managerial Insights}

In this paper, we have considered the question of how an assembler can cope with information asymmetry regarding her suppliers' marginal costs, particularly in settings with high demand uncertainty. We also considered the question of when using more complex contracting mechanisms, rather than simpler and easier to implement mechanisms, can add the most value for the assembler. To answer these questions, we compared three potential mechanisms, the FRW, hybrid, and ICDS mechanisms, where the latter two mechanisms are developed in this paper. We first characterized 
the optimal incentive compatible in dominant strategies (ICDS) mechanism for the assembler, under which the contracts offered to one supplier depend on all of the suppliers' types. We demonstrated how the assembler can compute the optimal ICDS contracts and we proved that the optimal procurement quantities are balanced, i.e., the same quantity is ordered from each supplier. We then compared the ICDS mechanism to the FRW mechanism, which requires the contracts offered to each supplier be a function only of that supplier's type, and thus is simpler to compute and implement. We found that the ICDS mechanism can significantly outperform the FRW mechanism, particularly when (1) there is significant uncertainty in the suppliers' costs, (2) there is significant uncertainty in the final product demand, and (3) the probability that a supplier is low-cost is large.

Further analysis of the FRW mechanism demonstrated that, for realistic problem sizes (i.e., with a moderate to large number of suppliers), the optimal FRW mechanism is identical to the naïve mechanism, under which the assembler assumes that all suppliers are high-cost and offers simple wholesale price contracts based on that assumption. Therefore, the results obtained in this paper clearly indicate that, in many realistic problem instances, the FRW mechanism, unlike the ICDS mechanism, cannot be used by the assembler to eliminate any of the inefficiency caused by information asymmetry regarding the suppliers' costs.

We also compared the ICDS and FRW mechanisms to a newly proposed hybrid mechanism. In developing the hybrid mechanism, we were motivated by the observation that, when the number of suppliers is moderate to large, the ICDS mechanism becomes complex to implement, while the FRW mechanism offers poor performance. Thus, the hybrid mechanism was designed to offer improved performance relative to the FRW mechanism, while being easier to implement than the ICDS mechanism, particularly when the number of suppliers is moderate to large. In such a setting, not all suppliers (or components) are of equal importance to, or have equal impact on, the assembler. For example, in the optimal ICDS mechanism, the rent paid by the assembler to a given supplier is increasing with the level of uncertainty regarding that supplier's cost, while the assembler's profit is decreasing in this uncertainty. Thus, in the hybrid mechanism, we measure the importance of a given supplier based on the degree of uncertainty the assembler has regarding that supplier's cost. The hybrid mechanism then uses the ICDS mechanism for a subset of suppliers with high uncertainty and the simpler naïve mechanism for the remaining suppliers with low uncertainty. 
From our numerical experiments, we found that the hybrid mechanism can achieve a performance close to that of the ICDS mechanism and can significantly outperform the FRW mechanism. However, we found that increasing the degree of sophistication of the contract mechanism (i.e., increasing the value of $k$ in the hybrid mechanism) is particularly beneficial in environments in which the assembler faces significant uncertainty regarding the end-customer demand and the suppliers' costs. In addition, we find that using the hybrid mechanism, rather than the FRW mechanism, is particularly important when (1) there is significant uncertainty in the suppliers' costs, (2) the marginal probability that a supplier is high-cost is small, (3) the number of suppliers is large, and (4) there is significant uncertainty in the final product demand. We also find that using the optimal ICDS mechanism, rather than the hybrid mechanism, is most critical when (1) the uncertainty regarding the suppliers' costs is large, (2) the assembler's expected profit margin is small, and (3) demand uncertainty is significant.

This final observation highlights a contribution of this paper relative to the economics literature. In the economics literature, there are numerous articles that consider the supplier's pricing game when the buyer possesses private demand information. In contrast, we consider a setting in which the suppliers possess private cost information, while the assembler faces uncertain final product demand. Among the economics articles that consider private cost information at an upstream stage, we are not aware of any that explicitly consider the impact of demand uncertainty at the downstream buyer. However, as we demonstrate in this paper, the magnitude of that demand uncertainty can have a significant impact on the value of using more sophisticated contract forms, such as the ICDS mechanism, relative to simpler mechanisms. In particular, when demand uncertainty is significant, it becomes critical for the buyer to apply a mechanism in which the contract parameters are dependent on all suppliers' types, so that she is able to make use of as much information as possible regarding the suppliers' costs when setting the order quantity.

Overall, our results demonstrate that in complex settings, such as those with a large number of suppliers or significant uncertainty, simple mechanisms, such as the FRW mechanism, can be almost completely ineffective in extracting cost information from the suppliers. In such settings, more sophisticated mechanisms, such as the hybrid and ICDS mechanisms, are necessary for the assembler to successfully cope with such information asymmetry. Furthermore, the additional 
complexity of the ICDS and hybrid mechanisms enables balanced ordering (and reduced waste) due to the fact that the order quantities are (potentially) a function of all of the suppliers' types, rather than a single supplier type, as in the FRW mechanism. Thus, a key insight obtained from this research is that while simplicity is of practical importance, in complex settings with information asymmetry, it should not be the only objective in mechanism design.

\section{References}

Bagnoli, M., T. Bergstorm. 2005. Log-concavity probability and its applications. Economic Theory. 26(2): 445-469.

Bergemann, D., S. Morris. 2005. Robust Mechanism Design. Econometrica. 73(6): 1771-1813.

Bergemann, D., J. Välimäki. 2006. Information in Mechanism Design. In Advances in Economics and Econometrics: Theory and Applications. R. Blundell, W. K. Newey, and T. Persson, editors. Volume 1, Chapter 5, Pages 186-221. Cambridge University Press, Cambridge, UK.

Chaturvedi, A., V. Martínez-de-Albéniz. 2011. Optimal procurement design in the presence of supply risk. Manufacturing $\&$ Service Operations Management. 13(2): 227-243.

Chung, K.-S., K. Ely. 2007. Foundations of Dominant-Strategy Mechanisms. Review of Economic Studies. 74(2): 447-476.

Fang, X., J. Ru, Y. Wang. 2014. Optimal procurement design of an assembly supply chain with information asymmetry. Production and Operations Management. 23(12): 2075-2088.

Hu, B., A. Qi. 2017. Optimal Procurement Mechanisms for Assembly. Available at SSRN: https://ssrn.com/abstract=2635069 or http://dx.doi.org/10.2139/ssrn.2635069. Accessed April $30,2018$.

Jehiel, P., M. Meyer-Ter-Vehn, B. Moldovanu, W.R. Zame. 2006. The Limits of Ex Post Implementation. Econometrica. 74(3): 585-610.

Kayis, E., F. Erhun, E. Plambeck. 2013. Delegation vs control of component procurement under asymmetric cost information and simple contracts. Manufacturing $\&$ Service Operations Management. 15(1): 45-56.

Levy, E., S. Ferazani. 2006. Standard \& Poor's industry survey: Autos and auto parts. Standard Poor's. 174(51): 1-39.

Melumad, N., D. Mookherjee, S. Reichelstein. 1995. Hierarchical decentralization of incentive contracts. RAND Journal of Economics. 26(4): 654-672.

Myerson, R. 1979. Incentive-compatibility and the bargaining problem. Econometrica. 47(1): 61-73. 
Vohra, R.V. 2012. Optimization and mechanism design. Mathematical Programming. 134(1): 283-303.

Wilson, R. 1987. Game-Theoretic Approaches to Trading Processes. T. Bewley, ed. Advances in Economic Theory: Fifth World Congress. Cambridge University Press, Cambridge, Chapter 2, 33-77.

Yang, Z., G. Aydin, V. Babich, D. Beil. 2012. Using a dual-sourcing option in the presence of asymmetric information about supplier reliability: Competition vs. diversification. Manufacturing \& Service Operations Management. 14(2): 202-217. 